\title{
THE LENGTHS OF PROJECTIVE TRIPLY-EVEN BINARY CODES
}

\author{
THOMAS HONOLD, MICHAEL KIERMAIER, SASCHA KURZ, AND ALFRED WASSERMANN
}

\begin{abstract}
It is shown that there does not exist a binary projective triply-even code of length 59 . This settles the last open length for projective triply-even binary codes. Therefore, projective triply-even binary codes exist precisely for lengths $15,16,30,31,32,45-51$, and $\geq 60$.
\end{abstract}

Keywords: divisible codes, projective codes, partial spreads MSC: Primary 94B05; Secondary 51E23.

\section{INTRODUCTION}

Doubly-even codes were subject to extensive research in the last years. For applications and enumeration results we refer e.g. to [6]. More recently, triply-even codes where studied, see e.g. [1]. These two classes of binary linear codes are special cases of so-called $\Delta$-divisible codes, where all weights of a $q$-ary linear code $\mathcal{C}$ are divisible by $\Delta$, see e.g. [13]. The columns of a $k \times n$ generator matrix of $\mathcal{C}$ generate $n$ one-dimensional subspaces of $\mathbb{F}_{q}^{k}$ that are also called points in Projective Geometry, see e.g. [5] or [3. Chapter 17]. The $\Delta$-divisibility of the linear code $\mathcal{C}$ translates as follows to the multiset $\mathcal{P}$ of points in $\mathbb{F}_{q}^{k}$. For each hyperplane $H$ of $\mathbb{F}_{q}^{k}$ we have $\#(\mathcal{P} \cap H) \equiv \# \mathcal{P}(\bmod \Delta)$. The set $\mathcal{P}$ is also called $\Delta$-divisible then. We remark that the hyperplanes correspond to the codewords of $\mathcal{C}$. A recent application of $\Delta$-divisible codes and sets is the maximum possible cardinality of partial $k$-spreads in $\mathbb{F}_{q}^{v}$, i.e., sets of $k$-dimensional subspaces in $\mathbb{F}_{q}^{v}$ with pairwise trivial intersection, see e.g. [7, 9]. Due to the intersection property, every point of $\mathbb{F}_{q}^{v}$ is covered by at most one element of a given partial $k$-spread. Calling every non-covered point a hole, we can state that the set of holes of a partial $k$-spread is $q^{k-1}$-divisible, see e.g. [7, Theorem 8] containing also a generalization to so-called vector space partitions ${ }^{1}$ So, from the non-existence of $q^{k-1}$-divisible sets (or projective $q^{k-1}$ divisible linear codes, since we have a set of holes in this application) of a suitable effective length $n$ one can conclude the non-existence of partial $k$-spreads in $\mathbb{F}_{q}^{v}$ of a certain cardinality. Indeed, all currently known upper bounds for partial $k$-spreads can be obtained from such non-existence results for divisible codes, see e.g. [7, 9].

From an application point of view, $q^{r}$-divisible linear codes, where $r$ is some positive rational number, are of special interest. If $G_{1}$ is a generator matrix of a $\Delta$-divisible $\left[n_{1}, k_{1}\right]_{q}$ code and $G_{2}$ is the generator matrix of another $\Delta$-divisible $\left[n_{2}, k_{2}\right]_{q}$ code, then $\left(\begin{array}{cc}G_{1} & 0 \\ 0 & G_{2}\end{array}\right)$ is the generator matrix of a $\Delta$-divisible $\left[n_{1}+n_{2}, k_{1}+k_{2}\right]_{q}$ code. Since the set of all points of a $k$-dimensional subspace of $\mathbb{F}_{q}^{v}$ is $q^{k-1}$-divisible, for each prime power $q$ and each $r \in \mathbb{Q}_{>0}$, such that $q^{r} \in \mathbb{N}$, there exists a finite set $\mathcal{F}_{q}(r)$ of integers that cannot be the cardinality of a $q^{r}$-divisible (multi)-set or effective length of a (projective) $q^{r}$-divisible linear code. For multisets of points, i.e., linear codes, the question is completely resolved in $[8$, Theorem 4] for all integers $r$ and all prime powers $q$. For sets of points or projective $q^{r}$-divisible linear codes the question is more complicated. A partial answer has been given in [7, Theorem 13]:

\section{Theorem 1.1.}

(i) $2^{1}$-divisible sets over $\mathbb{F}_{2}$ of cardinality $n$ exist for all $n \geq 3$ and do not exist for $n \in\{1,2\}$.

\footnotetext{
${ }^{1}$ In a special case, the divisibility of the set of holes was already used in [2] to determine an upper bound for the maximum cardinality of a partial $k$-spread.
} 
(ii) $2^{2}$-divisible sets over $\mathbb{F}_{2}$ of cardinality $n$ exist for $n \in\{7,8\}$ and all $n \geq 14$, and do not exist in all other cases.

(iii) $2^{3}$-divisible sets over $\mathbb{F}_{2}$ of cardinality n exist for $n \in\{15,16,30,31,32,45,46,47,48,49,50,51\}$, for all $n \geq 60$, and possibly for $n=59$; in all other cases they do not exist.

In part (iii) the existence question for a binary projective $2^{3}$-divisible linear code remains undecided. The aim of this paper is to complete this characterization result. Indeed, we will show that $n=59$ is impossible. We remark that the distinction between the existence of projective and possibly nonprojective $q^{r}$-divisible linear codes of a certain length plays indeed a role for e.g. upper bounds on the maximum possible cardinality of partial $k$-spreads. As an example, in [7, Theorem 13], see also [9], it is shown that no projective $2^{3}$-divisible linear code of length 52 exists, while there are non-projective examples with these parameters. From this non-existence result for projective $q^{r}$-divisible codes we can conclude that there can be at most 132 solids in $\mathbb{F}_{2}^{11}$ with pairwise trivial intersection, which is the tightest currently known upper bound. With a lower bound of 129, this is the smallest open case for the maximum cardinality of partial $k$-spreads over $\mathbb{F}_{2}$.

The remaining part of the paper is structured as follows. In Section 2 we state the necessary preliminaries from coding theory before we prove the non-existence of a binary projective $2^{3}$-divisible linear code with effective length $n=59$ in Section 3 . We close with a brief conclusion and some open problems in Section 4

\section{PRELIMINARIES}

A projective linear code $\mathcal{C}$ over $\mathbb{F}_{q}$ is called $q^{r}$-divisible for some $r \in \mathbb{Q}_{>0}$, such that $q^{r} \in \mathbb{N}^{2}$ if the weight of each codeword is divisible by $q^{r}$. By $A_{i}[\mathcal{C}]$ we denote the number of codewords of weight exactly $i$. Note that $A_{0}[\mathcal{C}]=1$. Whenever the code is clear from the context, we write $A_{i}$ instead of $A_{i}[\mathcal{C}]$. By $n=n(\mathcal{C})$ we denote the length and by $k=k(\mathcal{C})$ the dimension of the linear code. Given our assumption that $\mathcal{C}$ is projective the length equals the effective length ${ }^{3}$, i.e., there are no zero-columns in the generator matrix of $\mathcal{C}$ and we have a one-to-one correspondence to a set of $n$ spanning points in $\mathbb{F}_{q}^{k}$. Without creating confusion we use the same symbol $\mathcal{C}$ also for sets of points. By $a_{i}$ we denote the number of hyperplanes of $\mathbb{F}_{q}^{k}$ containing exactly $i$ points. Here, we have the relation $a_{i}=A_{n-i}$ for $0 \leq i<n$. Denoting the dual of a code by $\mathcal{C}^{\perp}$ we write $B_{i}=B_{i}(\mathcal{C})$ for the number of codewords of weight $i$ of $\mathcal{C}^{\perp}$. Due to our assumption that $\mathcal{C}$ is a projective code we have $B_{0}=1$ and $B_{1}=B_{2}=0$. The well-known MacWilliams identities, see e.g. [10], relate the $A_{i}$ with the $B_{i}$ as follows:

$$
\sum_{j=0}^{n} K_{i}(j) A_{j}(\mathcal{C})=2^{k} B_{i}(\mathcal{C}) \text { for } 0 \leq i \leq n,
$$

where

$$
K_{i}(j)=\sum_{s=0}^{n}(-1)^{s}\left(\begin{array}{c}
n-j \\
i-s
\end{array}\right)\left(\begin{array}{l}
j \\
s
\end{array}\right) \quad \text { for } 0 \leq i \leq n .
$$

Obviously, we have $\sum_{i=0}^{n} A_{i}=2^{k}$, which is indeed equivalent to the first $(i=0)$ MacWilliams equation. The polynomial $w(\mathcal{C})=\sum_{i=0}^{n} A_{i}(\mathcal{C}) x^{i}$ is called the weight enumerator of $\mathcal{C}$.

For a given $[n, k]_{q}$ code $\mathcal{C}$ and a codeword $c \in \mathcal{C}$ of weight $w$ we can consider the so-called residual code $\mathcal{C}_{w}$, which arises from $\mathcal{C}$ by restricting all codewords to those coordinates where $c$ has a zero entry. Thus, $\mathcal{C}_{w}$ is an $[n-w, \leq k-1]_{q}$ code. If $\mathcal{C}$ is projective, then obviously also $\mathcal{C}_{w}$ is projective. Moreover,

\footnotetext{
${ }^{2}$ More precisely, this conditions says that $q^{r}$ should be a power of the field characteristic $p$. In [11. Theorem 1] it has been shown that $\Delta$-divisible codes where $\Delta$ is relatively prime to $q$ correspond to repetitions of smaller codes. Thus, it suffices to consider $\Delta=p^{l}$ for integers $l$.

${ }^{3}$ Whenever we speak of the length of a code in this paper, we mean its effective length. So, $[n, k]_{q}$ codes are $k$-dimensional linear codes in $\mathbb{F}_{q}^{n}$ with effective length $n$.
} 
if $\mathcal{C}$ is $q^{r}$-divisible, then $\mathcal{C}_{w}$ is $q^{r-1}$-divisible, see e.g. [7, Lemma 7]. Since the possible lengths of binary projective $2^{2}$-divisible linear codes are characterized in Theorem 1.1.(ii), we can state that the weights of a binary projective $2^{3}$-divisible linear code $\mathcal{C}_{59}$ of length $n=59$ have to be contained in $\{8,16,24,32,40\}$. For codewords of weight 40 it is possible to characterize the possible corresponding residual codes:

Lemma 2.1. Let $\mathcal{C}$ be a projective $2^{2}$-divisible code of length $n=19$, then its weight enumerator is one of the following:

- $w(\mathcal{C})=1+1 \cdot x^{4}+75 \cdot x^{8}+51 \cdot x^{12}$, i.e., $k=7$ and $B_{3}=5$;

- $w(\mathcal{C})=1+78 \cdot x^{8}+48 \cdot x^{12}+1 \cdot x^{16}$, i.e., $k=7$ and $B_{3}=1$;

- $w(\mathcal{C})=1+4 \cdot x^{4}+150 \cdot x^{8}+100 \cdot x^{12}+1 \cdot x^{16}$, i.e., $k=8$ and $B_{3}=1$.

PROOF. The first four MacWilliams identities directly yield the three mentioned cases and additionally the weight enumerator $w(\mathcal{C})=1+5 \cdot x^{4}+147 \cdot x^{8}+103 \cdot x^{12}$, i.e., $k=8$ (and $B_{3}=3$ ). Using the MacWilliams transform we compute $B_{4}=2$. This contradicts $A_{4} \leq B_{4}$, which is implied by selforthogonality of $2^{2}$-divisible codes.

Moreover, each of the three mentioned weight enumerators is attained by a projective $2^{2}$-divisible code of length $n=19$ having a nice geometric description:

Assume that $\mathcal{P}$ is a 4-divisible set of points of size $n$ in some ambient $\mathbb{F}_{2}$-vector space $V$. For each plane $E$ intersecting $\mathcal{P}$ in a line $L, \mathcal{P}^{\prime}=(\mathcal{P} \backslash L) \cup(E \backslash L)$ is a 4-divisible set of points of size $n+1$. In other words, the line $L$ is switched to the affine plane $E \backslash L$. The 4-divisibility is seen by considering the characteristic functions of the involved point sets, see [7, Lemma 14]: We have $\chi_{\mathcal{P}^{\prime}}=\chi_{\mathcal{P}}+\chi_{E}-2 \chi_{L}$, where $\mathcal{P}$ and $E$ are 4-divisible and $L$ is 2-divisible.

In fact, all three types of projective 4-divisible codes of length 19 can be constructed by switching four pairwise skew lines $L_{1}, \ldots, L_{4}$ in a solid $S$. The choice of $L_{1}, \ldots, L_{4}$ is unique up to isomorphism and arises as the unique line spread of $S$ without one of its lines. In particular, each code is the union of the line $S \backslash\left\{L_{1}, L_{2}, L_{3}, L_{4}\right\}$ and 4 affine planes. For the explicit constructions below, we pick $L_{1}, L_{2}, L_{3}$ and $L_{4}$ as the row spaces of the following matrices:

$$
L_{1}:\left(\begin{array}{cccc}
1 & 0 & 0 & 0 \\
0 & 1 & 0 & 0
\end{array}\right) \quad L_{2}:\left(\begin{array}{cccc}
1 & 0 & 1 & 0 \\
0 & 1 & 0 & 1
\end{array}\right) \quad L_{3}:\left(\begin{array}{cccc}
1 & 0 & 1 & 1 \\
0 & 1 & 1 & 0
\end{array}\right) \quad L_{4}:\left(\begin{array}{cccc}
1 & 0 & 0 & 1 \\
0 & 1 & 1 & 1
\end{array}\right)
$$

The remaining three points of $S$ are the points on the line

$$
\left(\begin{array}{llll}
0 & 0 & 1 & 0 \\
0 & 0 & 0 & 1
\end{array}\right)
$$

The lines will be embedded in the ambient space $\left(\mathbb{F}_{2}^{7}\right.$ or $\left.\mathbb{F}_{2}^{8}\right)$ by adding all-zero coordinates. Furthermore, the $i$ th unit vector will be denoted by $e_{i}$ and for a vector $v$, the point generated by $v$ will be denoted by $\langle v\rangle$.

By the uniqueness of the choice of the 4 lines, the result $\mathcal{P}$ of the switching only depends on its image in $V / S$ of size 4 . There are 3 possible constellations of 4 points:

- Four points in general position, spanning a solid. The resulting code $C_{1}$ is of dimension 8 and has weight distribution $\left(0^{1} 4^{4} 8^{150} 12^{100} 16^{1}\right)$ and 18432 automorphisms.

For an explicit construction, we switch in the planes $L_{1}+\left\langle e_{5}\right\rangle, L_{2}+\left\langle e_{6}\right\rangle, L_{3}+\left\langle e_{7}\right\rangle$ and $L_{4}+\left\langle e_{8}\right\rangle$. This leads to the generator matrix

$$
\left(\begin{array}{lllllllllllllllllll}
0 & 0 & 0 & 0 & 1 & 0 & 1 & 0 & 1 & 0 & 1 & 0 & 1 & 0 & 1 & 0 & 1 & 0 & 1 \\
0 & 0 & 0 & 0 & 0 & 1 & 1 & 0 & 0 & 1 & 1 & 0 & 0 & 1 & 1 & 0 & 0 & 1 & 1 \\
1 & 0 & 1 & 0 & 0 & 0 & 0 & 0 & 1 & 0 & 1 & 0 & 1 & 1 & 0 & 0 & 0 & 1 & 1 \\
0 & 1 & 1 & 0 & 0 & 0 & 0 & 0 & 0 & 1 & 1 & 0 & 1 & 0 & 1 & 0 & 1 & 1 & 0 \\
0 & 0 & 0 & 1 & 1 & 1 & 1 & 0 & 0 & 0 & 0 & 0 & 0 & 0 & 0 & 0 & 0 & 0 & 0 \\
0 & 0 & 0 & 0 & 0 & 0 & 0 & 1 & 1 & 1 & 1 & 0 & 0 & 0 & 0 & 0 & 0 & 0 & 0 \\
0 & 0 & 0 & 0 & 0 & 0 & 0 & 0 & 0 & 0 & 0 & 1 & 1 & 1 & 1 & 0 & 0 & 0 & 0 \\
0 & 0 & 0 & 0 & 0 & 0 & 0 & 0 & 0 & 0 & 0 & 0 & 0 & 0 & 0 & 1 & 1 & 1 & 1
\end{array}\right) .
$$


- The three points on a line together with an additional point (i.e., the complement of a triangle in a plane). The resulting code $C_{2}$ is of dimension 7 and has weight distribution $\left(0^{1} 4^{1} 8^{75} 12^{51}\right)$ and 1440 automorphisms.

Switching in the planes $L_{1}+\left\langle e_{5}\right\rangle, L_{2}+\left\langle e_{6}\right\rangle, L_{3}+\left\langle e_{7}\right\rangle, L_{4}+\left\langle e_{6}+e_{7}\right\rangle$, we get the explicit generator matrix

$$
\left(\begin{array}{lllllllllllllllllll}
0 & 0 & 0 & 0 & 1 & 0 & 1 & 0 & 1 & 0 & 1 & 0 & 1 & 0 & 1 & 0 & 1 & 0 & 1 \\
0 & 0 & 0 & 0 & 0 & 1 & 1 & 0 & 0 & 1 & 1 & 0 & 0 & 1 & 1 & 0 & 0 & 1 & 1 \\
1 & 0 & 1 & 0 & 0 & 0 & 0 & 0 & 1 & 0 & 1 & 0 & 1 & 1 & 0 & 0 & 0 & 1 & 1 \\
0 & 1 & 1 & 0 & 0 & 0 & 0 & 0 & 0 & 1 & 1 & 0 & 1 & 0 & 1 & 0 & 1 & 1 & 0 \\
0 & 0 & 0 & 1 & 1 & 1 & 1 & 0 & 0 & 0 & 0 & 0 & 0 & 0 & 0 & 0 & 0 & 0 & 0 \\
0 & 0 & 0 & 0 & 0 & 0 & 0 & 1 & 1 & 1 & 1 & 0 & 0 & 0 & 0 & 1 & 1 & 1 & 1 \\
0 & 0 & 0 & 0 & 0 & 0 & 0 & 0 & 0 & 0 & 0 & 1 & 1 & 1 & 1 & 1 & 1 & 1 & 1
\end{array}\right) .
$$

The following alternative construction is worth noting: Switching three pairwise disjoint lines in $S$ such that the image modulo $S$ is a line results in a binary 4-divisible [18,6]-code. This code is the same as the concatenation of the $\mathbb{F}_{4}$-linear hexacode (arising from a hyperoval over $\mathbb{F}_{4}$ ) with the binary $[3,2]$ simplex code. Geometrically, it is the disjoint union of 6 lines in an ambient space $H$ of algebraic dimension 6 . Switching any of its lines always increases the ambient space dimension by one and leads to the code $C_{2}$. The hyperplane $H$ corresponds $18-3=15$ points and belongs to the single codeword of weight 4 .

- An affine plane (i.e., the complement of a line in a plane). The resulting code $C_{3}$ is of dimension 7 and has weight distribution $\left(0^{1} 8^{78} 12^{48} 16^{1}\right)$ and 5760 automorphisms.

Switching in the planes $L_{1}+\left\langle e_{5}\right\rangle, L_{2}+\left\langle e_{6}\right\rangle, L_{3}+\left\langle e_{7}\right\rangle, L_{4}+\left\langle e_{5}+e_{6}+e_{7}\right\rangle$, we get the explicit generator matrix

$$
\left(\begin{array}{lllllllllllllllllll}
0 & 0 & 0 & 0 & 1 & 0 & 1 & 0 & 1 & 0 & 1 & 0 & 1 & 0 & 1 & 0 & 1 & 0 & 1 \\
0 & 0 & 0 & 0 & 0 & 1 & 1 & 0 & 0 & 1 & 1 & 0 & 0 & 1 & 1 & 0 & 0 & 1 & 1 \\
1 & 0 & 1 & 0 & 0 & 0 & 0 & 0 & 1 & 0 & 1 & 0 & 1 & 1 & 0 & 0 & 0 & 1 & 1 \\
0 & 1 & 1 & 0 & 0 & 0 & 0 & 0 & 0 & 1 & 1 & 0 & 1 & 0 & 1 & 0 & 1 & 1 & 0 \\
0 & 0 & 0 & 1 & 1 & 1 & 1 & 0 & 0 & 0 & 0 & 0 & 0 & 0 & 0 & 1 & 1 & 1 & 1 \\
0 & 0 & 0 & 0 & 0 & 0 & 0 & 1 & 1 & 1 & 1 & 0 & 0 & 0 & 0 & 1 & 1 & 1 & 1 \\
0 & 0 & 0 & 0 & 0 & 0 & 0 & 0 & 0 & 0 & 0 & 1 & 1 & 1 & 1 & 1 & 1 & 1 & 1
\end{array}\right) .
$$

The code $C_{3}$ also arises from shortening the $[24,12]$ extended binary Golay code 5 times. Note that the result does not depend on the choice of the 5 positions, as the automorphism group of the Golay code (the Mathieu group $M_{24}$ ) acts 5 -fold transitive on set of the coordinates.

\section{THE NON-EXISTENCE OF BINARY PROJECTIVE $2^{3}$-DIVISIBLE LINEAR CODES OF LENGTH $n=59$}

Setting $y=2^{k}$, the first four MacWilliams identities yield

$$
\begin{aligned}
& A_{16}[\mathcal{C}]=-10-4 A_{8}-\frac{45}{4096} y+\frac{1}{4096} y B_{3} \\
& A_{24}[\mathcal{C}]=20+6 A_{8}+\frac{1447}{4096} y-\frac{3}{4096} y B_{3} \\
& A_{32}[\mathcal{C}]=-15-4 A_{8}+\frac{2617}{4096} y+\frac{3}{4096} y B_{3} \\
& A_{40}[\mathcal{C}]=4+A_{8}+\frac{77}{4096} y-\frac{1}{4096} y B_{3} .
\end{aligned}
$$

Thus, we have $A_{16}+A_{40}=-6-3 A_{8}+\frac{y}{128} \geq 0$, so that $y \geq 768$. (Commonly, we will write just $A_{i}$ instead of $A_{i}[\mathcal{C}]$.) Since $y=2^{k}$, we conclude $k \geq 10$ and $y \geq 1024$. Choosing a fixed dimension $k$, the 
non-negativity constraints for the $A_{i}$ and $B_{i}$ form a polyhedron of dimension at most 2, i.e., a polygon. As parameterizing variables, we use $A_{8}$ and $B_{3}$. Since $A_{16} \geq 0$, we have

$$
B_{3} \geq 45+\frac{10 \cdot 4096}{y} .
$$

Since $A_{16}+4 A_{40} \geq 0$, we have

$$
B_{3} \leq 87+\frac{2}{3}+\frac{2^{13}}{y}
$$

so that

$$
A_{8}+A_{16} \leq \frac{42+\frac{2}{3}}{4096} y-8
$$

Since $A_{16}+A_{40} \geq 0$, we have

$$
A_{8} \leq \frac{y}{384}-2
$$

Definition 3.1. Let $\mathcal{C}$ be a projective linear code of effective length $n$ in $\mathbb{F}_{q}^{n}$ with dimension $k=\operatorname{dim}(\mathcal{C})>$ 2. For a pair of different codewords (of $\mathcal{C}$ ) let $\mathcal{T}:=\left\langle c_{1}, c_{2}\right\rangle=\left\{c_{1}, c_{2}, c_{3}\right\}$ denote the two-dimensional subcode generated by $c_{1}$ and $c_{2}$. By $\left(\left\{w\left(c_{1}\right), w\left(c_{2}\right), w\left(c_{3}\right)\right\}, \frac{w\left(c_{1}\right)+w\left(c_{2}\right)+w\left(c_{3}\right)}{2}\right)$ we denote the subcodetype of $\mathcal{T}$ Here, the first three numbers, read as a multiset, are the weights of the codewords of $\mathcal{T}$ and the last number is the corresponding effective length.

As an example, we consider a binary projective $2^{3}$-divisible code $\mathcal{C}$ of length $n=59$ and list the possible subcode-types containing a fixed codeword of length 40 :

- $e_{1}:(\{40,24,16\}, 40)$;

- $e_{2}:(\{40,32,8\}, 40)$;

- $e_{3}:(\{40,24,24\}, 44)$

- $e_{4}:(\{40,32,16\}, 44)$;

- $e_{5}:(\{40,40,8\}, 44)$;

- $e_{6}:(\{40,32,24\}, 48)$

- $e_{7}:(\{40,40,16\}, 48)$;

- $e_{8}:(\{40,40,24\}, 52)$;

- $e_{9}:(\{40,32,32\}, 52)$;

- $e_{10}:(\{40,40,32\}, 56)$.

Here $e_{i}$ denotes the number of times this subcode-type occurs in $\mathcal{C}$, where a codeword of weight 40 is fixed. Counting the codewords of $\mathcal{C}$ by weight gives

$$
\begin{aligned}
A_{40}[\mathcal{C}] & =1+e_{5}+e_{7}+e_{8}+e_{10} \\
A_{32}[\mathcal{C}] & =e_{2}+e_{4}+e_{6}+2 e_{9}+e_{10} \\
A_{24}[\mathcal{C}] & =e_{1}+2 e_{3}+e_{6}+e_{8} \\
A_{16}[\mathcal{C}] & =e_{1}+e_{4}+e_{7} \\
A_{8}[\mathcal{C}] & =e_{2}+e_{5}
\end{aligned}
$$

so that obviously $\sum_{i=1}^{10} e_{i}=2^{\operatorname{dim}(\mathcal{C})-1}-1=2^{k-1}-1$. In the remaining part of this section $\mathcal{C}$ always denotes a binary projective $2^{3}$-divisible code of length $n=59$. 
Lemma 3.2. Let $\mathcal{D}$ be the residual code of a codeword cof weight 40 of . Setting $z=2^{\operatorname{dim}(\mathcal{C})-\operatorname{dim}(\mathcal{D})-1}$, we have

$$
\begin{aligned}
z-1 & =e_{1}+e_{2} \\
z \cdot A_{4}[\mathcal{D}] & =e_{3}+e_{4}+e_{5} \\
z \cdot A_{8}[\mathcal{D}] & =e_{6}+e_{7} \\
z \cdot A_{12}[\mathcal{D}] & =e_{8}+e_{9} \\
z \cdot A_{16}[\mathcal{D}] & =e_{10} .
\end{aligned}
$$

Proof. Observe that the non-zero weights of $\mathcal{D}$ are contained in $\{4,8,12,16\}$, since $\mathcal{D}$ is $2^{2}$-divisible, and count the 2 -dimensional subcodes of $\mathcal{C}$ containing codeword $c$ by their effective length.

Lemma 3.3. Let $\mathcal{C}$ be a projective $2^{3}$-divisible code of length $n=59, k=\operatorname{dim}(\mathcal{C})$ and $d$ be a codeword of weight 40 . For the corresponding residual code $\mathcal{D}$ we have $w(\mathcal{D}) \neq 1+78 \cdot x^{8}+48 \cdot x^{12}+1 \cdot x^{16}$.

Proof. Assume the contrary. Since $A_{16}[\mathcal{D}]=1$ and $\operatorname{dim}(\mathcal{D})=7$, we have $e_{10}=2^{k-8}$, so that $A_{40} \geq 2^{k-8}+1$. Since $e_{1}+e_{2}=2^{k-8}-1$, we have $A_{8}+A_{16} \geq 2^{k-8}-1$. Thus, $A_{8}+A_{16}+A_{40} \geq$ $2^{k-7}$. From the above we compute $A_{24}+A_{32}=5+2 A_{8}+\frac{127}{128} y=5+2 A_{8}+\frac{127}{128} \cdot 2^{k}$, so that $A_{8}+A_{16}+A_{24}+A_{32}+A_{40} \geq 5+2 A_{8}+2^{k}>2^{k}-1$, which is a contradiction.

Lemma 3.4. Let $\mathcal{C}$ be a projective $2^{3}$-divisible code of length $n=59, k=\operatorname{dim}(\mathcal{C})$ and d be a codeword of weight 40 . For the corresponding residual code $\mathcal{D}_{1}$ we have $w\left(\mathcal{D}_{1}\right) \neq 1+4 \cdot x^{4}+150 \cdot x^{8}+100 \cdot x^{12}+1 \cdot x^{16}$.

Proof. Assume the contrary. Since $A_{16}\left[\mathcal{D}_{1}\right]=1$ and $\operatorname{dim}\left(\mathcal{D}_{1}\right)=8$, we have $e_{10}=2^{k-9}$, so that $A_{40} \geq 2^{k-9}+1$. Since $e_{1}+e_{2}=2^{k-9}-1$, we have $A_{8}+A_{16} \geq 2^{k-9}-1$. Now we switch to the geometric language. By $\overline{\mathcal{C}}$ we denote the set of 59 points in $\mathbb{F}_{2}^{k}$ corresponding to $\mathcal{C}$ and by $\overline{\mathcal{D}}_{1}$ we denote the set of 19 points corresponding to $\mathcal{D}$, i.e., there exists a hyperplane $H_{1}$ of $\mathbb{F}_{2}^{k}$ with $\overline{\mathcal{D}}_{1}=H_{1} \cap \overline{\mathcal{C}}$. Since $e_{10} \geq 1$, there exists another hyperplane $H_{2}$ such that $\# \overline{\mathcal{D}}_{2}=19$ and $\# \overline{\mathcal{D}}_{1} \cap \overline{\mathcal{D}}_{2}=3$, where $\overline{\mathcal{D}}_{2}=H_{2} \cap \overline{\mathcal{C}} \cdot e_{10}=2^{k-9}$ counts the number of subspaces $S$ of codimension 2 of $\mathbb{F}_{2}^{k}$ with $S \leq H_{1}$ and $\# S \cap \overline{\mathcal{C}}=3$. One of these, call it $S^{\prime}$, is also contained in $H_{2}$, i.e., $S^{\prime}=H_{1} \cap H_{2}$. Let $\mathcal{D}_{2}$ denote the residual code corresponding to $\overline{\mathcal{D}}_{2}$. Since $S^{\prime} \cap \overline{\mathcal{C}}$ corresponds to a codeword of weight 16 in $\mathcal{D}_{2}$. Thus, $H_{2}$ contains $2^{k-1-\operatorname{dim}\left(\mathcal{D}_{2}\right)} \geq 2^{k-9}$ subspaces $S$ of codimension 2 of $\mathbb{F}_{2}^{k}$ with $S \leq H_{2}$ and $\# S \cap \overline{\mathcal{C}}=3$. In total we have at least $2^{k-8}-1$ different subspaces $S_{i}$ of codimension 2 of $\mathbb{F}_{2}^{k}$ with $\# S \cap \overline{\mathcal{C}}=3$ and either $S \leq H_{1}$ or $S \leq H_{2}$. ( $H_{1} \cap H_{2}$ determines a unique such subspace.) Let us number these subspaces in such a way that $S_{1}=H_{1} \cap H_{2}, S_{i} \leq H_{1}, S_{i} \not \leq H_{2}$ for $2 \leq i \leq 2^{k-9}$ and $S_{i} \leq H_{2}, S_{i} \not \leq H_{1}$ for $2^{k-9}+1 \leq i \leq 2^{k-8}-1$. Each of these subspaces $S_{i}$ determines two unique hyperplanes $H_{i, 1}$ and $H_{i, 2}$ of $\mathbb{F}_{2}^{k}$ with $S_{i} \leq H_{i, j}$ and $\# H_{i, j} \cap \overline{\mathcal{C}}=19$. W.1.o.g. we assume $H_{1,1}=H_{1}, H_{1,2}=H_{2}, H_{1,1}=H_{1}$ for $2 \leq i \leq 2^{k-9}$ and $H_{i, 1}=H_{2}$ for $2^{k-9}+1 \leq i \leq 2^{k-8}-1$. With this, the hyperplanes $H_{1}, H_{2}$, and $H_{i, 2}$ for $2 \leq i \leq 2^{k-8}-1$ are pairwise disjoint by construction and contain exactly 19 points of $\overline{\mathcal{C}}$ each. Thus, we have $A_{40}[\mathcal{C}] \geq 2^{k-8}$.

Let $H_{1}$ and $H_{2}$ be the same two hyperplanes as above. Since $e_{1}+e_{2}=2^{k-9}-1$, there are $2^{k-9}-1$ subspaces $T$ of codimension 2 of $\mathbb{F}_{2}^{k}$ with $T \leq H_{1}$ and $\# T \cap \overline{\mathcal{C}}=19$. Since $\operatorname{dim}\left(\mathcal{D}_{2}\right) \leq 8$ there are (at least) $2^{k-9}-1$ subspaces $T$ of codimension 2 of $\mathbb{F}_{2}^{k}$ with $T \leq H_{2}$ and $\# T \cap \overline{\mathcal{C}}=19$. Since $\# \overline{\mathcal{D}}_{1} \cap \overline{\mathcal{D}}_{2}=3$, we have at least $2^{k-8}-2$ such subspaces $T_{1}, \ldots, T_{2^{k-8}-2}$. Each of these subspaces is either contained in a hyperplane containing 43 or 51 points of $\overline{\mathcal{C}}$. For $1 \leq i, j \leq 2^{k-8}-2$ and $i \neq j$ assume that there is a hyperplane $H$ of $\mathbb{F}_{2}^{k}$ with $T_{i} \leq H, T_{j} \leq H$, and $\# H \cap \overline{\mathcal{C}} \geq 4$. W.1.o.g. we assume $T_{i} \leq H_{1}$ and $T_{j} \leq H_{2}$. (Observe that $\operatorname{dim}\left(T_{i} \cup T_{j}\right)=k-1$, so that e.g. $S_{i} \leq H_{1}$ and $S_{j} \leq H_{1}$ implies $\left\langle S_{i}, S_{j}\right\rangle=H_{1}=H$.) Now, we argue that $\# H \cap \overline{\mathcal{C}}=51$. Assume, to the contrary, that $\# H \cap \overline{\mathcal{C}}=43$. Since $\# \overline{\mathcal{D}}_{1}=19, \# \overline{\mathcal{D}}_{2}=19$, and $\# \overline{\mathcal{D}}_{1} \cap \overline{\mathcal{D}}_{2}=3$, we have $\# \overline{\mathcal{C}}^{\prime}=32$, where $\overline{\mathcal{C}}^{\prime}=\overline{\mathcal{D}}_{1} \cup \overline{\mathcal{D}}_{2}-\overline{\mathcal{D}}_{1} \cap \overline{\mathcal{D}}_{2}$. Note that $\overline{\mathcal{C}}^{\prime}$ is a $2^{2}$-divisible set, so that $H \cap \overline{\mathcal{C}}-\overline{\mathcal{C}}^{\prime}$ is also a $2^{2}$-divisible set. However, there is no 
$2^{2}$-divisible set of cardinality $43-32=11$, which is a contradiction. Thus, $A_{16} \geq 2 \cdot\left(2^{k-9}-1\right)-2 A_{8}$, which can be rewritten to $2 A_{8}+A_{16} \geq 2^{k-8}-2$. Thus, we have

$$
-6-A_{8}+2^{k-7}=2 A_{8}+A_{16}+A_{40} \geq 2^{k-8}-2+2^{k-8}=2^{k-7}-2,
$$

so that $A_{8} \leq-4$, which is a contradiction.

Lemma 3.5. There is no projective $2^{3}$-divisible code of length $n=59$, dimension $k \leq 10$ that contains a codeword of weight 40.

Proof. As shown at the beginning of this section, we have $k \geq 10$, so that we can assume $k=10$. Now, assume the contrary and let $\mathcal{D}$ be the residual code of a codeword of weight 40 . Due to Lemma 2.1 and Lemma 3.4 we have $\operatorname{dim}(\mathcal{D})=7$. Since $e_{1}+e_{2}=2^{k-8}-1$, we have $A_{8}+A_{16} \geq 2^{k-8}-1$. However, this contradicts $A_{8}+A_{16} \leq \frac{42+\frac{2}{3}}{4096} y-8=\frac{8}{3}<3$, which was concluded above from the first four MacWilliams identities.

Lemma 3.6. If there is projective $2^{3}$-divisible code of length $n=59$, then there also exists such a code with dimension $k=10$.

Proof. Let $\mathcal{C}$ be a projective $2^{3}$-divisible code of length $n=59$ with minimum possible dimension $k$ and $\mathcal{P}$ be the corresponding set of points in $\mathbb{F}_{2}^{k}$, i.e., the points spanned by the columns of an arbitrary generator matrix of $\mathcal{C}$. For each point $Q$ in $\mathbb{F}_{2}^{k}$ that is not contained in $\mathcal{C}$ we consider the projection through $Q$, that is the multiset image of $\mathcal{P}$ under the map $\mathbb{F}_{2}^{k} \rightarrow \mathbb{F}_{2}^{k} / Q, v \mapsto(v+Q) / Q$. The result is a multiset of points in $\mathbb{F}_{2}^{k-1}$ where all points on a common line through $Q$ got identified. The corresponding linear code $\mathcal{C}^{\prime}$ is a subcode of $\mathcal{C}$ and therefore a $2^{3}$-divisible binary linear code of (effective) length $n=59$, since $Q$ is not contained in $\mathcal{P}$, and dimension $k-1$. The code $\mathcal{C}^{\prime}$ is non-projective iff every point $Q$ not contained in $\mathcal{P}$ lies on a secant, i.e., on a line consisting of $Q$ and two points in $\mathcal{P}$. Due to the assumed minimality of $k$, any point of $\mathbb{F}_{2}^{k} \backslash \mathcal{P}$ must lie on a secant. $\mathcal{P}$ admits at most $\left(\begin{array}{c}\# \mathcal{P} \\ 2\end{array}\right)=\left(\begin{array}{c}59 \\ 2\end{array}\right)=1711$ secants which cover at most 1711 different points not in $\mathcal{P}$. On the other hand, there are $2^{k}-60$ points not contained in $\mathcal{P}$, which forces $k \leq 10$. As shown at the beginning of this section, we have $k \geq 10$, so that $k=10$.

Theorem 3.7. There is no binary projective $2^{3}$-divisible linear code of effective length 59.

Proof. Assume that $\mathcal{C}$ is a binary projective $2^{3}$-divisible linear code of effective length $n=59$. Due to Lemma 3.6 we can assume that $\mathcal{C}$ has dimension $k=10$. As mentioned in Section 2 , the weights are contained in $\{8,16,24,32,40\}$. Weight 40 is excluded in Lemma 3.5. Plugging $A_{40}[\mathcal{C}]=0$ and $y=2^{10}$ into the equations at the beginning of this section gives $A_{16}[\mathcal{C}]=2-3 A_{8}[\mathcal{C}]$, where $A_{8}[\mathcal{C}]$ is considered as the free parameter in the solution of the first four MacWilliams identities. Since $A_{8}[\mathcal{C}]$ and $A_{16}[\mathcal{C}]$ are non-negative integers, we conclude that $A_{8}[\mathcal{C}]=0$ and obtain the unique solution $A_{16}[\mathcal{C}]=2$, $A_{24}[\mathcal{C}]=312, A_{32}[\mathcal{C}]=709$.

Now let $c$ be one of the two codewords of weight 16 and $\mathcal{C}^{\prime}$ be the restriction of $\mathcal{C}$ to $\operatorname{supp}(c)$, i.e., to the 16 non-zero positions of $c$. The code $\mathcal{C}^{\prime}$ is a binary linear code of effective length 16 . By the $2^{3}$-divisibility of $\mathcal{C}$ and the fact that $\mathcal{C}^{\prime}$ contains the all-1-word, we see that $\mathcal{C}^{\prime}$ is $2^{2}$-divisible.

Consider a codeword $x \in \mathcal{C}$ whose restriction to $\operatorname{supp}(c)$ is the zero word. Then $w(x+c)=w(x)+$ $16 \leq 32$ and therefore $w(x) \leq 16$. Thus, $x$ is either the zero word of $\mathcal{C}$ or, possibly, the second word of weight 16 . This implies $\operatorname{dim}\left(\mathcal{C}^{\prime}\right) \geq k-1=9 \sqrt[4]{4}$ However, the $2^{2}$-divisibility implies that $\mathcal{C}^{\prime}$ is self-orthogonal of length 16 and therefore of dimension at most $16 / 2=8$-contradiction.

\footnotetext{
${ }^{4}$ To make this argument precise, apply the rank-nullity theorem to the surjective linear map $\varphi: \mathcal{C} \rightarrow \mathcal{C}^{\prime}$ which restricts a codeword to the 16 coordinates in $\operatorname{supp}(c)$.
} 


\section{CONCLUSION AND OPEN PROBLEMS}

By purely theoretical methods we were able to exclude the existence of a binary projective $2^{3}$-divisible linear code of effective length $n=59$. This completes the characterization of the possible lengths of $2^{3}$ divisible projective linear codes, which play some role in applications.

We might have streamlined our theoretical reasoning. Lemma 3.3 may be removed completely and the proof of Lemma 3.4 may be restricted to the case $k=10$. However, we have included their full proofs due to the subsequent reason. In Lemma 3.3 we have used a codeword of weight 40 to conclude the existence of a certain number of codewords of weight at most 16 that contradicts the MacWilliams identities. In Lemma 3.4 we have used a refined analysis starting from two codewords of weight 40 . In principle each codeword of weight 40 implies the existence of some codewords of weight at most 16 . Since there have to be a lot of codewords of weight 40, there should also be quite some codewords of weight at most 16 . It would be very nice to turn this vague idea into a rigorous theoretic argument for the exclusion of the third possible weight enumerator in Lemma 2.1

While we prefer a theoretical argument over computer computations, we nevertheless state an alternative computational verification of our main result in the appendix. The computational methods might also be applicable for other coding theoretic existence questions.

Clearly, it would be desirable to see generalizations of the (completed) characterization Theorem 1.1 for other parameters. To this end, we state the list of length of $2^{4}$-divisible binary projective linear codes for which the existence question is undecided, at least up to our knowledge:

$$
\{130,163,164,165,185,215,216,232,233,244,245,246,247,274,275,277,278,306,309\} \text {. }
$$

For $q=3$ the smallest open case is that of $3^{2}$-divisible ternary projective linear codes with

$$
\{70,77,99,100,101,102,113,114,115,128\}
$$

as the set of undecided lengths.

\section{ACKNOWLEDGMENTS}

We would like to thank Iliya Bouyukliev for discussions on the usage of his software Q-Extension and a personalized version that is capable to deal with larger dimensions, see the appendix.

\section{REFERENCES}

[1] K. Betsumiya and A. Munemasa. On triply even binary codes. Journal of the London Mathematical Society, 86(1):1-16, 2012.

[2] A. Beutelspacher. Partial spreads in finite projective spaces and partial designs. Mathematische Zeitschrift, 145(3):211-229, 1975.

[3] J. Bierbrauer. Introduction to coding theory. Chapman and Hall/CRC, 2004.

[4] I. Bouyukliev. What is Q-extension? Serdica Journal of Computing, 1(2):115-130, 2007.

[5] S. Dodunekov and J. Simonis. Codes and projective multisets. The Electronic Journal of Combinatorics, 5(1):37, 1998.

[6] C. F. Doran, M. G. Faux, S. J. Gates, T. Hübsch, K. M. Iga, G. D. Landweber, and R. L. Miller. Codes and supersymmetry in one dimension. Advances in Theoretical and Mathematical Physics, 15(6):1909-1970, 2011.

[7] T. Honold, M. Kiermaier, and S. Kurz. Partial spreads and vector space partitions. In Network Coding and Subspace Designs, pages 131-170. Springer, 2018.

[8] M. Kiermaier and S. Kurz. An improvement of the Johnson bound for subspace codes. arXiv preprint 1707.00650, 2017.

[9] S. Kurz. Packing vector spaces into vector spaces. The Australasian Journal of Combinatorics, 68(1):122-130, 2017.

[10] F. J. MacWilliams and N. J. A. Sloane. The theory of error-correcting codes. Elsevier, 1977.

[11] H. N. Ward. Divisible codes. Archiv der Mathematik, 36(1):485-494, 1981.

[12] H. N. Ward. A bound for divisible codes. IEEE Transactions on Information Theory, 38(1):191-194, 1992.

[13] H. N. Ward. An introduction to divisible codes. Designs, Codes and Cryptography, 17(1):73-79, 1999. 


\section{ApPENDiX A. An ALternative COMPUTATIONAL APPROACH}

Instead of the theoretical reasoning presented in Section 3 , Theorem 3.7 can also be obtained by computer calculations. Our starting point is a computational version of Lemma 2.1] In [6] binary projective $2^{2}$-divisible linear codes with small parameters were classified using extensive computer enumerations. For length 19 there are exactly three non-isomorphic examples, see https://rlmill.github.io/ de_codes, which of course match our three geometric constructions given in Section 2 . We have verified the specific case $n=19$ using $\mathrm{Q}-$ Extension [4].

\begin{tabular}{rrrrrrrrrrrrrrrrrrrr}
\hline $\mathrm{k} / \mathrm{n}$ & 8 & 12 & 14 & 15 & 16 & 20 & 22 & 23 & 24 & 26 & 27 & 28 & 29 & 30 & 31 & 32 & 34 & 35 & 36 \\
\hline 1 & 1 & 0 & 0 & 0 & 1 & 0 & 0 & 0 & 1 & 0 & 0 & 0 & 0 & 0 & 0 & 1 & 0 & 0 & 0 \\
2 & & 1 & 0 & 0 & 1 & 1 & 0 & 0 & 2 & 0 & 0 & 2 & 0 & 0 & 0 & 3 & 0 & 0 & 3 \\
3 & & & 1 & 0 & 1 & 1 & 1 & 0 & 3 & 1 & 0 & 4 & 0 & 3 & 0 & 8 & 2 & 0 & 9 \\
4 & & & & 1 & 1 & 1 & 1 & 1 & 4 & 1 & 1 & 6 & 1 & 6 & 4 & 18 & 7 & 3 & 27 \\
5 & & & & 1 & 0 & 0 & 1 & 4 & 2 & 1 & 7 & 1 & 8 & 8 & 32 & 14 & 7 & 54 \\
6 & & & & & & & & 1 & 0 & 1 & 6 & 2 & 7 & 8 & 34 & 11 & 7 & 65 \\
7 & & & & & & & & & & & 1 & 1 & 6 & 6 & 24 & 5 & 3 & 36 \\
8 & & & & & & & & & & & & & 2 & 4 & 13 & 1 & 1 & 11 \\
9 & & & & & & & & & & & & & & 1 & 5 & 0 & 0 & 1 \\
10 & & & & & & & & & & & & & & & \\
\hline
\end{tabular}

TABLE 1 . Number of $[n, k]_{2}$ codes with weights in $\{8,16,24,32,40\}-$ part 1 .

The next step is to show that none of the three mentioned codes above can be a residual code of a binary projective $2^{3}$-divisible code of length $n=59$. Next we provide all computational results for the case of the weight enumerator $w(\mathcal{C})=1+1 \cdot x^{4}+75 \cdot x^{8}+51 \cdot x^{12}$. First note that the unique isomorphism type can be represented by the following generator matrix:

$$
M_{19}=\left(\begin{array}{l}
1000001111000110100 \\
0100001110010101010 \\
0010001100110011100 \\
0001000101101010110 \\
0000101001100111010 \\
0000011001100101110 \\
0000001010001011111
\end{array}\right)
$$

With this, the generator matrix of a $2^{3}$-divisible projective linear code $C_{59}$ of length $n=59$, that contains a codeword of weight 40 , can be written as

$$
\left(\begin{array}{cc}
M_{19} & M_{1} \\
0 & M_{2}
\end{array}\right),
$$

where $M_{2}$ is the generator matrix of a $2^{3}$-divisible linear code $C_{40}$ of length $n=40$ that is not necessarily projective. Using the software package $Q-E x t e n s i o n$ [4] we have enumerated all binary linear codes with effective length $n \leq 40$ and weights in $\{8,16,24,32,40\}$, see Table 1 and Table 2 Here blank entries on the left hand side of each row as well as missing columns correspond to effective lengths where no such code exists. We remark that the $2^{3}$-divisible linear codes up to length $n=48$ have been determined, in principle, in [1]. We have validated our results with the corresponding list of codes at http://www.st.hirosaki-u.ac.jp/ betsumi/triply-even/.

Thus, the dimension of $C_{40}$ is at most 11 , so that the dimension of $C_{59}$ is at most 18 . We remark that the divisible code bound from [12] gives an upper bound of 21 for the dimension. In order to enumerate the possibilities for $C_{59}$ we can start from one of the codes $C_{40}$ and iteratively add one row to 


\begin{tabular}{rrrrr}
\hline $\mathrm{k} / \mathrm{n}$ & 37 & 38 & 39 & 40 \\
\hline 1 & 0 & 0 & 0 & 1 \\
2 & 0 & 0 & 0 & 4 \\
3 & 0 & 6 & 0 & 17 \\
4 & 2 & 22 & 10 & 64 \\
5 & 5 & 59 & 36 & 194 \\
6 & 8 & 79 & 57 & 347 \\
7 & 5 & 61 & 49 & 323 \\
8 & 1 & 21 & 30 & 177 \\
9 & 0 & 2 & 10 & 59 \\
10 & 0 & 0 & 1 & 11 \\
11 & 0 & 0 & 0 & 1
\end{tabular}

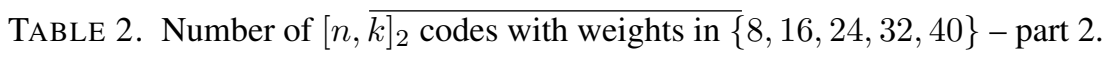

the generator matrix maintaining the property that the code is $2^{3}$-divisible. We start by formulating this general approach as an enumeration problem of integral points in a polyhedron:

Lemma A.1. Let $G$ be a systematic generator matrix of an $[n, k]_{2}$ code whose weights are $\Delta$-divisible and are contained in $[a \cdot \Delta, b \cdot \Delta]$. By $c(u)$ we denote the number of columns of $G$ that equal $u$ for all $u$ in $\mathbb{F}_{2}^{k} \backslash \mathbf{0}, c(\mathbf{0})=n^{\prime}-n$, and let $\mathcal{S}(G)$ be the set of feasible solutions of

$$
\begin{aligned}
\Delta y_{h}+\sum_{v \in \mathbb{F}_{2}^{k+1}: v^{\top} h=0} x_{v}=n-a \Delta & \forall h \in \mathbb{F}_{2}^{k+1} \backslash \mathbf{0} \\
x_{(u, 0)}+x_{(u, 1)}=c(u) & \forall u \in \mathbb{F}_{2}^{k} \\
x_{e_{i}} \geq 1 & \forall 1 \leq i \leq k+1 \\
x_{v} \in \mathbb{N} & \forall v \in \mathbb{F}_{2}^{k+1} \\
y_{h} \in\{0, \ldots, b-a\} & \forall h \in \mathbb{F}_{2}^{k+1} \backslash \mathbf{0},
\end{aligned}
$$

where $e_{i}$ denotes the ith unit vector in $\mathbb{F}_{2}^{k+1}$ and $n^{\prime} \geq n+1$. Then, for every systematic generator matrix $G^{\prime}$ of an $\left[n^{\prime}, k+1\right]_{2}$ code $C^{\prime}$ whose first $k$ rows coincide with $G$ we have a solution $(x, y) \in \mathcal{S}(G)$ such that $G^{\prime}$ has exactly $x_{v}$ columns equal to $v$ for each $v \in \mathbb{F}_{2}^{k+1}$.

Proof. Let such a systematic generator matrix $G^{\prime}$ be given and $x_{v}$ denote the number of columns of $G^{\prime}$ that equal $v$ for all $v \in \mathbb{F}_{2}^{k+1}$. Since $G^{\prime}$ is systematic, Equation 3 is satisfied. As $G^{\prime}$ arises by appending a row to $G$, also Equation (2) is satisfied. Obviously, the $x_{v}$ are non-negative integers. The conditions (1) and $(5)$ correspond to the restriction that the weights are $\Delta$-divisible and contained in $\{a \Delta, \ldots, b \Delta\}$.

We remark that also every solution in $\mathcal{S}(G)$ corresponds to an $\left[n^{\prime}, k+1\right]_{2}$ code $C^{\prime}$ with generator matrix $G^{\prime}$ containing $C$ as a subcode. Different generator matrices of corresponding isomorphic codes may be reduced to just one representing generator matrix using the tools from Q-Extension [4]. In order to implement our extra knowledge of the generator matrix $M_{19}$ of the residual code, we ensure that the extended generator matrices coincide outside of the 40 columns of $C_{40}$ with the last $r$ rows of $M_{19}$, where $r$ increases from 1 to 7 . In terms of the integer linear program from Lemma A.1 we just fix the counts $x_{v}$ of the corresponding columns $v$. At $r=7$ we can check if the resulting codes are projective. It turns out that this never happens, so that:

Lemma A.2. Let $\mathcal{C}$ be a projective $2^{3}$-divisible code of length $n=59, k=\operatorname{dim}(\mathcal{C})$ and $d$ be a codeword of weight 40 . For the corresponding residual code $\mathcal{D}$ we have $w(\mathcal{D}) \neq 1+1 \cdot x^{4}+75 \cdot x^{8}+51 \cdot x^{12}$. 
Without giving the computational details, we remark that we have performed the described computational approach also for the two other residual codes, so that we obtain:

Corollary A.3. Let $\mathcal{C}$ be a projective $2^{3}$-divisible code of length $n=59$. Then all non-zero weights of $\mathcal{C}$ are contained in $\{8,16,24,32\}$.

We remark that we might have used inequalities coming from the MacWilliams identities, like $A_{8}+$ $A_{16} \leq \frac{42+\frac{2}{3}}{4096} y-8$, to cut the generation tree of linear codes. I.e. at every point where we have constructed a new subcode, we may count the number of codewords $A_{8}$ and $A_{16}$ of weight 8 and 16 , respectively. The number of codewords of weight at most 16 of the final code of length $n=59$ cannot decrease. The starting code $C_{40}$ also fixes the dimension of the final code to $k=\operatorname{dim}\left(C_{40}\right)+7$, so that we may check the violation of the above inequality using $y=2^{k}$. However, the computer enumerations were fast enough so that it was not necessary to implement these checks.

\begin{tabular}{rrrrrrrrrrrr}
\hline $\mathrm{k} / \mathrm{n}$ & 40 & 41 & 42 & 43 & 44 & 45 & 46 & 47 & 48 & 49 & 50 \\
\hline 2 & 2 & 0 & 0 & 0 & 1 & 0 & 0 & 0 & 1 & 0 & 0 \\
3 & 11 & 0 & 6 & 0 & 9 & 0 & 5 & 0 & 6 & 0 & 3 \\
4 & 49 & 2 & 36 & 12 & 62 & 9 & 50 & 15 & 59 & 9 & 42 \\
5 & 154 & 12 & 158 & 67 & 316 & 62 & 362 & 146 & 503 & 135 & 495 \\
6 & 277 & 29 & 335 & 180 & 903 & 273 & 1394 & 704 & 2533 & 877 & 3245 \\
7 & 241 & 26 & 356 & 215 & 1194 & 528 & 2663 & 1829 & 6038 & 2176 & 8341 \\
8 & 119 & 12 & 176 & 147 & 738 & 513 & 2285 & 2534 & 6753 & 2443 & 6643 \\
9 & 29 & 3 & 49 & 57 & 244 & 257 & 1000 & 1610 & 3535 & 1416 & 1876 \\
10 & 4 & 0 & 3 & 12 & 41 & 54 & 229 & 499 & 144 & 480 & 50 \\
11 & & & & 1 & 3 & 14 & 51 & 90 & 98 & 69 & 36 \\
12 & & & & & 2 & 8 & 14 & 7 & 15 & 4 \\
13 & & & & & & 1 & 1 & 0 & 1 & 0 \\
\hline
\end{tabular}

At this point we know if $\mathcal{C}_{59}$ is a projective $2^{3}$-divisible code of length $n=59$, then its weights are upper bounded by 32 . So we enumerate those linear codes, that are possibly non-projective, using Q-Extension [4]. Of course, for $n<40$ the counts coincide with those from Table 1 and Table 2]. For length $n \geq 40$ we state the resulting counts in Table 3 and Table 4

Having checked that all resulting codes of length $n=59$ are non-projective we conclude our main result Theorem 3.7

We remark that the divisible code bound from [12] gives an upper bound of 17 for binary linear codes with weights in $\{8,16,24,32\}$.

Thomas Honold, ZJU-UIUC Institute, ZheJiAng University, 314400 Haining, China.

Email address: honolde z ju. edu. cn

Michael Kiermaier, University of Bayreuth, 95440 Bayreuth, Germany

Email address: michael.kiermaier@uni-bayreuth.de

SASCHA KURZ, UNIVERSITY OF BAYREUTH, 95440 BAYREUTH, GERMANY

Email address: sascha.kurz@uni-bayreuth. de

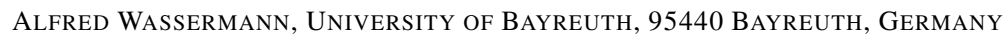

Email address: alfred.wassermann@uni-bayreuth. de 


\begin{tabular}{rrrrrrrrrr}
\hline $\mathrm{k} / \mathrm{n}$ & 51 & 52 & 53 & 54 & 55 & 56 & 57 & 58 & 59 \\
\hline 2 & 0 & 0 & 0 & 0 & 0 & 0 & 0 & 0 & 0 \\
3 & 0 & 2 & 0 & 1 & 0 & 1 & 0 & 0 & 0 \\
4 & 16 & 30 & 10 & 15 & 7 & 7 & 4 & 2 & 1 \\
5 & 210 & 444 & 206 & 291 & 157 & 144 & 74 & 40 & 17 \\
6 & 1713 & 3695 & 2252 & 3160 & 2523 & 2295 & 1585 & 886 & 334 \\
7 & 4585 & 10523 & 7026 & 9634 & 10043 & 11322 & 10393 & 8195 & 3695 \\
8 & 3877 & 7309 & 5860 & 6852 & 9477 & 12719 & 14811 & 15536 & 9237 \\
9 & 1238 & 1643 & 1975 & 2132 & 3655 & 6134 & 7659 & 8871 & 6965 \\
10 & 198 & 171 & 305 & 224 & 768 & 547 & 558 & 911 & 2905 \\
11 & 10 & 16 & 48 & 65 & 69 & 259 & 204 & 295 & 675 \\
12 & 1 & 1 & 6 & 10 & 7 & 31 & 48 & 56 & 174 \\
13 & 0 & 0 & 1 & 1 & 0 & 3 & 7 & 8 & 34 \\
14 & & & & & & 1 & 1 & 6 \\
15 & \multicolumn{1}{c}{ TABLE 4. Number of $n n, k]_{2}$ codes with weights in $\{8,16,24,32\}-$ part 2.}
\end{tabular}

